\title{
The Power Transformer Differential Protection Using Decision Tree
}

\author{
Javad AZARAKHSH \\ Faculty of Marine Engineering, Chabahar Maritime University, Chabahar, Iran \\ email address: j.azarakhsh@cmu.ac.ir
}

\begin{abstract}
This paper presents a new power transformer protection method by intelligent differential relay. This method is based on differential current sampling caused by primary and secondary three phase current of transformer. Detecting the type of fault in protection place is based on performance of the learned decision tree. The simulations of power system and differential relay are accomplished in PSCAD and MATLAB. The main characteristic of the relay is being intelligence such that at first step the decision tree is learned by several samples including faulted and inrush currents and then the relay detects the internal fault intelligently and quickly and the outputs of the relay order to the breakers in two ends of the transformer to be opened.
\end{abstract}

Keywords: Transformer; Differential Relay; Wavelet Transform; Correlation Coefficient; On-line performance

\section{Introduction}

Today, many schemes have been considered by designers in order to increase the efficiency even slightly and also effective performance against the inrush and faulted currents due to high importance of differential protection. Because even these slight changes can lead to improve in total system. Therefore, the enhancement of efficiency by $\% 0.01$ is significantly valuable. Some of techniques have been proposed to improve the efficiency of differential relay are utilizing the means like time-frequency devices to analysis the differential signals and neural networks to get the system smart [1-5], [10,11].

The major contribution of the power equipment is transformer which is used in energy transmission lines. The damages caused by internal fault in any time and with any amplitude are considered as an important problem and the faulted transformer must be removed to protect the ocomponents of system. On the other hand, the differential protection procedure has to be accurate. So the differential protection is the most important type of protecting. Some of the disturbances cause to inaccurate performance in differential protection such as inrush current, transformer saturation and increasing the excitation current. Exact and fast fault detection and distinguishing between inrush current and internal fault is the most important aspect of differential protection, such that an incorrect closing order due to inaccurate detection can lead to irreparable economic damages.

Recently the wavelet transform is known as the basic method to extract the features of transient signals due to its capability to analysis these signals. The wavelet based methods can successfully be used to analysis various signals particularly the signals with transient nature and time variant signals like power system disturbances [1]. Some of the transformer protection methods have used the S-transform and neural networks [2]. The fuzzy logic is another used method [3]. An effective method to prevent the inaccurate performance of the differential relay against inrush current is the 
one mentioned in [4]. In this method, first the differential and robust current are calculated and the main frequency of each current is compared with the others in order to detect the phase angle difference (PAD) or the secondary currents of the related transformer. Another method is based on wavelet transform, neural networks or integrated wavelet transform based on support vector machine [7], [20]. In the other technique, the mean absolute deviation (MAD) and the wavelet coefficients on a specific frequency band of wavelet transform are used [8], [21]. A method which makes difference based on the differential current slope between inrush current and internal fault is presented in [9]. This simulation is based on the angle of the differential current slope vector in A, $\mathrm{B}$ and $\mathrm{C}$ phases throughout the sampling window. The inrush current is detectable by using of statistical computations since the vector behavior of this current is different in short circuit conditions. In some literatures the Clarke transform and discrete wavelet transform are used to distinguish between internal fault and the other cases [10]. Bayesian classification (BC) is another method to differential protect the transformer which operates base on rules in parallel with neural network [11]. Discrete Fourier transform with neural network are utilized to identify the inrush current and internal fault [12]. In [13] and [14] the concept of power differential is mentioned. The wavelet transform integrated with ANFIS is the other way to identify the inrush current and internal fault $[15,16]$. The proposed algorithm based on harmonic processing of the differential current to protect the transformer in contribution with ANN is presented in [17]. Adaptive differential protection [18] and also decision tree [19] are used to protect the transformer.

In this paper we use the decision tree method to off-line differential protect of the power transformer which is a sub-methods of the neural network. The decision tree is learned by several faulted and inrush current samples simulated by means of PSCAD and is tested by some stochastic current components in MATLAB environment.

The paper is organized as follow: the pattern recognition, decision tree and learning algorithm are presented in Sections II, III and IV respectively. The studied system in the paper is provided in Section V. The proposed method is described in Section VI. The simulation results and a comparison with the similar works are presented in Section VII and finally the conclusions are stated in Section VIII.

\section{Pattern recognition}

One of the existing phenomena detection methods is identifying based on features, but the features which provide the distinguishing possibility between the phenomena and the others. The configuration of the features and their changing manner is a behavior pattern for the phenomena which can be considered in order to recognize it. This is known as Pattern Recognition.

If the purpose of the pattern recognition methods is identifying the phenomena with stochastic behavior patterns and the ones whose behavior manner is changed randomly based on the conditions, then we cannot define an exact and certain pattern for behavior manner and decide based on. So the methods must be used in choosing the pattern recognition method which considers all of the behavior patterns of the phenomena as much as possible. One of these methods is neural network in which the behavior pattern of the phenomena is learned to be used as a criterion in recognition. The accurate distinguishing between the faulted and inrush currents requires a suitable choosing of pattern recognition method which finally leads to correct learning of the system. 
In this paper we extract the desired features of two current components by choosing the proper pattern that these features are the first and second harmonic components and distortion and wavelet transforms energy and then we explain the extraction procedure and how to use of each feature.

\section{Decision tree}

The decision tree algorithm is one of methods which are used to recognize the phenomena with uncertain features. This algorithm has a structure like neural network, but it is simpler and faster. In this structure it is usually learned a known behavior pattern to the decision tree through the learning algorithm in order to phenomena recognize which gives it the capability for clustering the similar phenomena.

One of the most important characteristics of decision tree is its capability to give priority to the parameters to distinguish between the behavior of purpose phenomena and the others who have similar behavior pattern. This always leads to obtain an optimal tree by considering the making decision time, learning time and the decision accuracy related to identify the intended pattern.

\section{Learning algotithm}

The decision tree algorithm must be learned by a desired method in order to be capable of choosing phenomena among the others. In this paper we use the following procedure to create the decision tree:

a) Extracting the purpose phenomena by considering the changes of one or more features (Since the capability of the decision tree in finding or giving priority to the features. It is better to be more the number of the measured features of the phenomena).

b) Extracting the behavior of the other phenomena which may interfere with the purpose phenomena (similar phenomena).

c) The learning of the decision tree is as follows:

1. Choosing the best feature to distinguish of the other similar phenomena based on their behavior sensitivity to the features changes (using the clustering coefficient). The most important choice in learning the decision tree in that which features must be choose as the basis for decision making. On the other words, the feature is proper for decision making (separating the clusters) that can cluster the input data. The clustering coefficient can measure the desire performance of each feature in separating the input samples based on purpose clusters.

The clustering coefficient is defined based on entropy concept. The entropy is an index that shows the amount of impurity of data toward the desired feature or illustrates the possibility of clustering the input data to the decision tree toward the desired feature. On the other words, if the impurity of dada is zero then there is only one class or cluster among data toward the desired feature and means that the entropy index is zero that equals to be zero the physical changes of data toward the desired feature.

The entropy of B set contains the input samples, is defined based on $\mathrm{n}$ different classes as follows:

$$
\operatorname{Entropy}(B)=\sum_{i=1}^{n}-p_{i} \log _{2} p_{i}
$$

where $p_{i}$ is the ratio of the number of members in a subset of B which placed in class $\mathrm{i}$. 
Now the clustering coefficient of data, $C F\left(B, A_{i j}\right)$, which could define the amount of proportionality of choosing feature $\mathrm{A}$ from the $\mathrm{B}$ as the distinction between behavior of purpose phenomena and the others as follows:

$$
C F\left(B, A_{i, j}\right)=\operatorname{Entropy}(B)-\sum_{V=1}^{n} \frac{\left|B_{V}\right|}{|B|} \operatorname{Entropy}\left(B_{V}\right)
$$

While $B_{V}$ is a subset of B such that desired $A_{i j}$ has the $b_{V}$ value (i is the number of sub-tree and $\mathrm{j}$ is the subcategory of tree in level i).

2. Executing the strategy of paragraph 1 in a lower level- As it shown in Figure 1, the algorithm in paragraph 1 is executed among the subsets of input data clustered based on various desired values of $\mathrm{B}$.

3. Expanding the tree in each path- This continues until all of data can be take in a cluster or class, on the other words the entropy of all data in subset $b_{V}$ be zero.

Changing the decision tree to the logic rules- The learned decision trees are ambiguous even in simplest form. This is because each node is the content of a specific value which is obtained by experiments in higher nodes. In order to be more readable the decision tree we can explain the path to each cluster by if-then rules. The if part contains all the experiments in the path and then part only contains the final cluster.

These rules are known as "logic rules" and these total rules can recognize the pattern of all the clusters in the decision tree framework.

\section{Typical system}

A typical single phase system is shown in Figure 1. In this study the PSCAD has been used to simulate the typical system. The power system in Figure 2 contains a three phase $230 \mathrm{kV}, 50 \mathrm{~Hz}$ power supply which feeds a resistive-inductive $100 \mathrm{MW}, 500 \mathrm{kVAr}$ load through a Y-Y 230/63, 300 MVA transformer. We use the "Three Phase Fault" block to simulate the internal fault. In next stage we apply this fault on different phases in various time intervals.

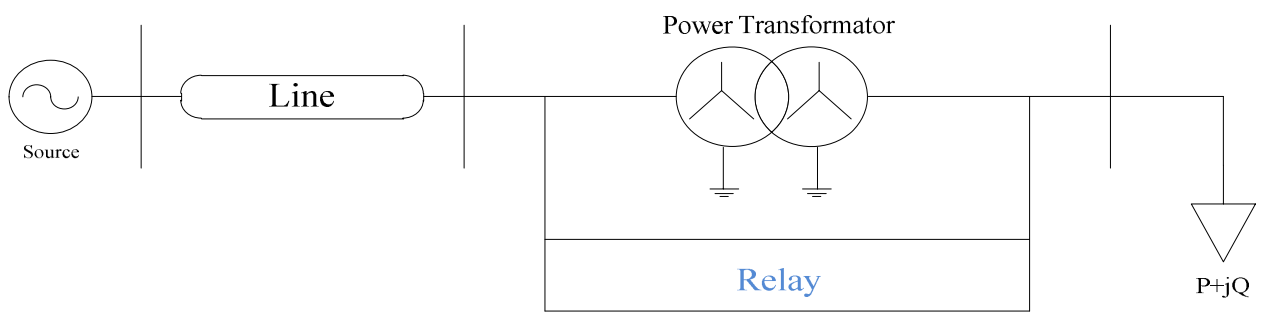

Fig. 1: The typical single phase system

The typical power system is illustrated in Figure 2. 


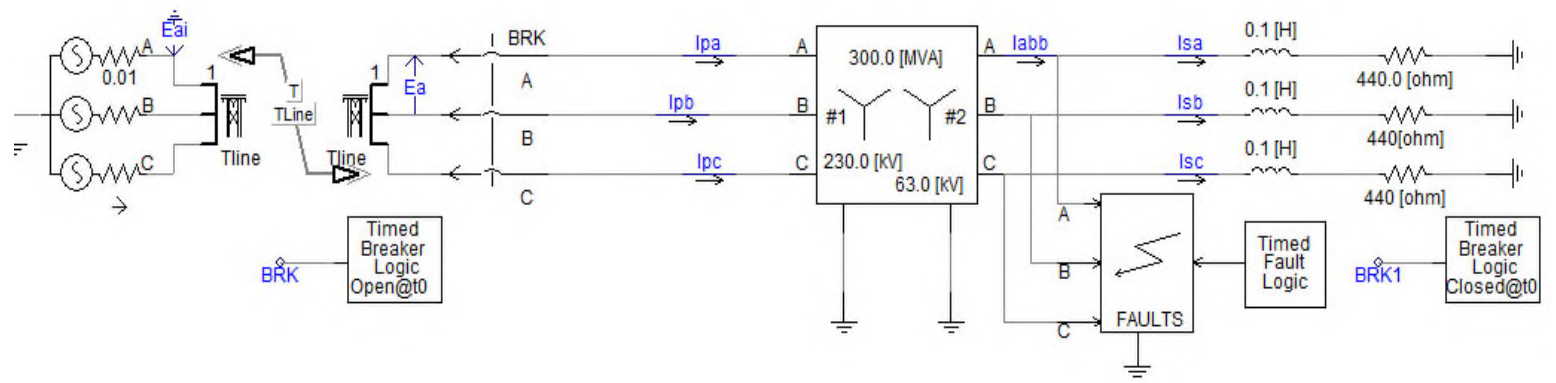

Fig. 2: The simulated power system

We use the hysteresis curve in Figure 3 to simulate the inrush current which leads to saturate the transformer at startup.

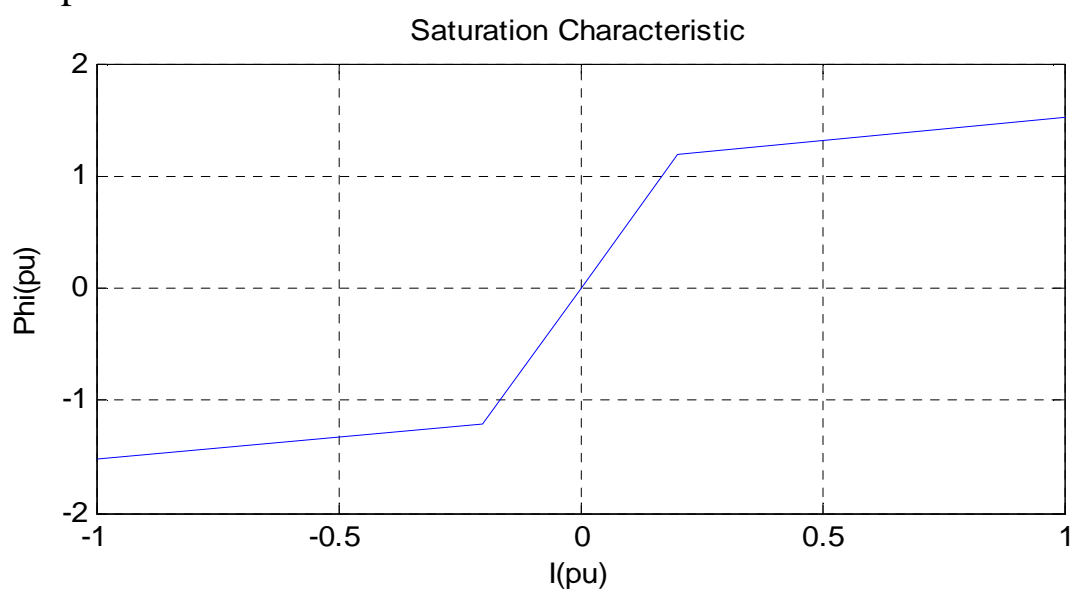

Fig. 3: The saturation characteristic of transformer

\section{The proposed method}

\subsection{The differential current forming}

The windings of three phase $\mathrm{Y}-\mathrm{Y}$ transformer can be built in $\Delta-\Delta$ form into the current transformer. Therefor the currents of differential relay are measured by two current transformers at two ends of power transformer and the differential algorithm of differential currents is calculated as equations (3) and (5).

$$
\begin{aligned}
& I_{1}=\left(I_{A P}-I_{A S}\right)-\left(I_{C P}-I_{C S}\right) \\
& I_{2}=\left(I_{B P}-I_{B S}\right)-\left(I_{A P}-I_{A S}\right) \\
& I_{3}=\left(I_{C P}-I_{C S}\right)-\left(I_{B P}-I_{B S}\right)
\end{aligned}
$$

The block diagram of differential currents procedure by means of MATLAB as the inputs to the relay is shown in Figure 4. 


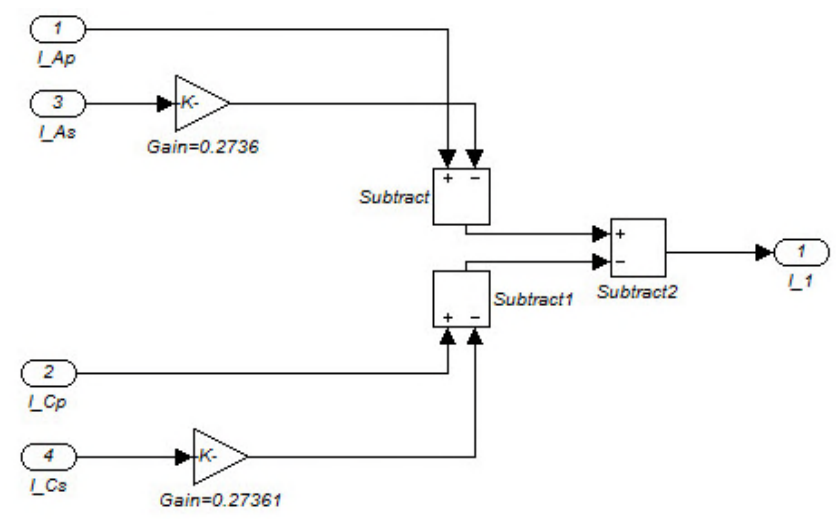

Fig. 4: The differential current forming algorithm

\subsection{Features extraction}

The features extraction and clustering is the most important steps among all steps of a tree forming or neural network learning since the desired performance of an intelligent system is defined based on type of the learned feature to the decision tree. On the other hand, the proper choosing and clustering of the features is equal to better performance of tree and the accuracy parameter of tree is close to 100 if the tree is properly and completely learned as we expect.

One of the clear characteristics of inrush current is that it has high, transient and time variant harmonic components while the faulted current has steady and single harmonic components. Using of applicable means in frequency domain will be helpful in order to extract the features related to the harmonic components of signals. The features used in this paper are: the energy of first harmonic, the energy of second harmonic, distortion and the energy of wavelet transform respectively which in the following we will explain the extraction procedure of each feature.

a) The energy of first and second harmonics: These signals have harmonic components so the energy of first and second harmonic components in different cases can affect the decision of tree. The flowcharts of Figs. 5 and 6 show the extraction procedure of these two features.

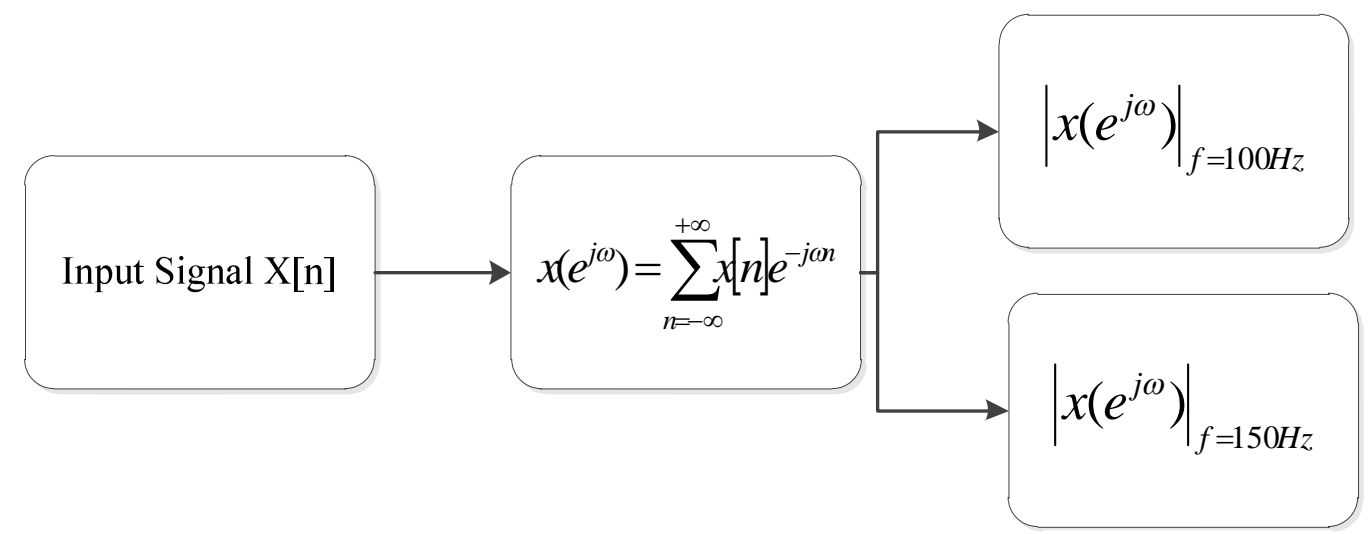

Fig. 5: The flowchart of extracting the energy of the first and second harmonics

b) Distortion: The equation (1) is used to calculate the amount of the signal distortion. 


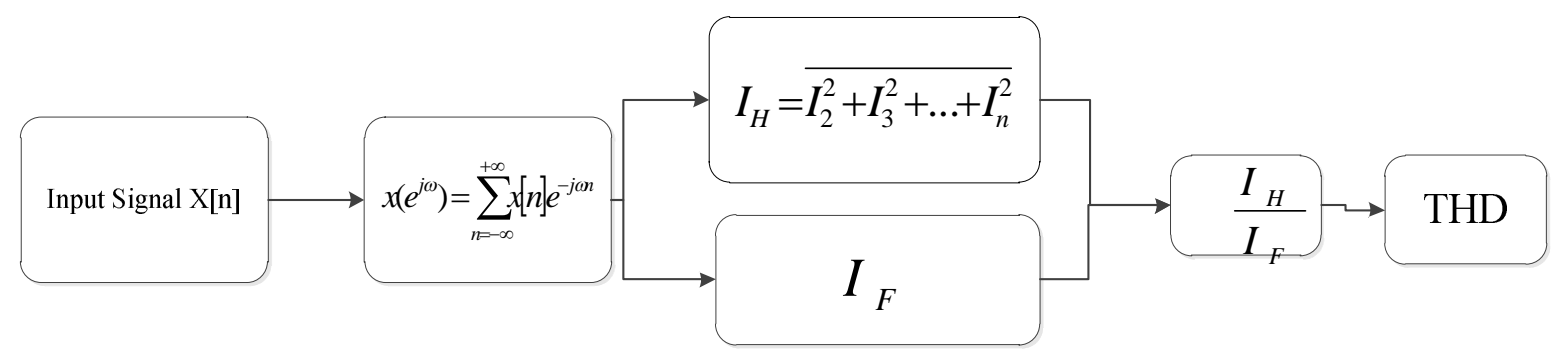

Fig. 6: The flowchart of THD extraction

c) The energy of wavelet transform: The Fast Fourier Transform (FST) is a tool which is applicable in specific time intervals and frequency and isn't suitable for inrush distortions analysis, but the wavelet transform is obtained from transmitting and changing the scale of a primary function named the mother wavelet function and it can create various intervals in different frequencies and it is suitable for time variant, transient and non-periodic signals. The wavelet is a waveform which has limited time interval and zero mean value.

The equation of discrete wavelet transform is as follows:

$$
c(a, b)=\int_{R} s(t) \frac{1}{\sqrt{a}} \Psi\left(\frac{t-b}{a}\right) d t
$$

where $s(t)$ is the signal that wavelet transform is extracted from it and $\Psi(t)$ is the mother wavelet and also:

$$
a=2^{j}, b=k 2^{j},(j, k) \in z^{2}
$$

where $j$ presents the decomposition level of signal and $k$ is time in discrete form. In fact $C(a, b)$ is a coefficient who shows the similarity between the transmitted and scaled mother wavelet and the basic signal.

The coefficients $A_{j}$ and $D_{j}$ are created in each level of wavelet transform as follows:

$$
\begin{gathered}
D_{j}(t)=\sum_{k \in z} c(j, k) \Psi_{j, k}(t) \\
\Psi_{j, k}(t)=2^{-j / 2} \Psi\left(2^{-j} t-k\right), j \in z, k \in z \\
A_{J}=\sum_{j \succ J} D_{j}
\end{gathered}
$$

$A_{j}$ is an approximation of the basic signal and $D_{j}$ shows the details of the signal.

There are two low-pass and high-pass filters in this transform and every wavelet has its own filter. As it can be seen in Figure 7, in the first step of signal $\mathrm{S}$ is recording two approximation coefficient $c A_{l}$ and detail coefficient $c D_{i}$ which these two vectors are created by convoluting the signal $\mathrm{S}$ to the filters. This means that convoluting the signal by low-pass filter $L o \_D$ and highpass filter $H i \_D$ creates the approximation and detail coefficients, respectively. 


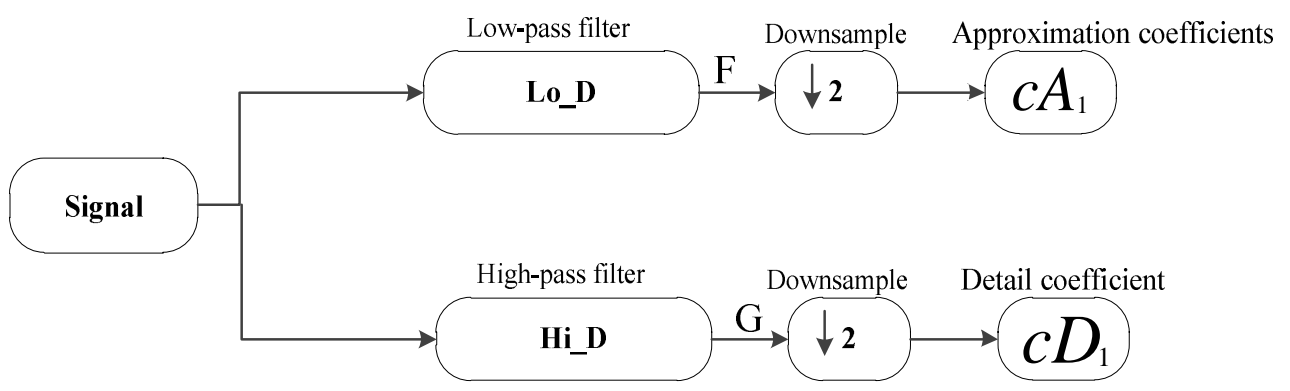

Fig. 7: Single level wavelet transform

\subsection{Determining the efficient feature in learning}

After extracting the four features of each signal we can determine the efficient feature which has the significant contribution in making decision and finally accurate learning of the tree in order to arrange the rules before applying these features to the tree. As it can be seen in Figure 8, the fourth feature has the more essential contribution in both inrush and faulted currents, since it has two separated forms for inrush and faulted currents, as is evident in Figure 8.

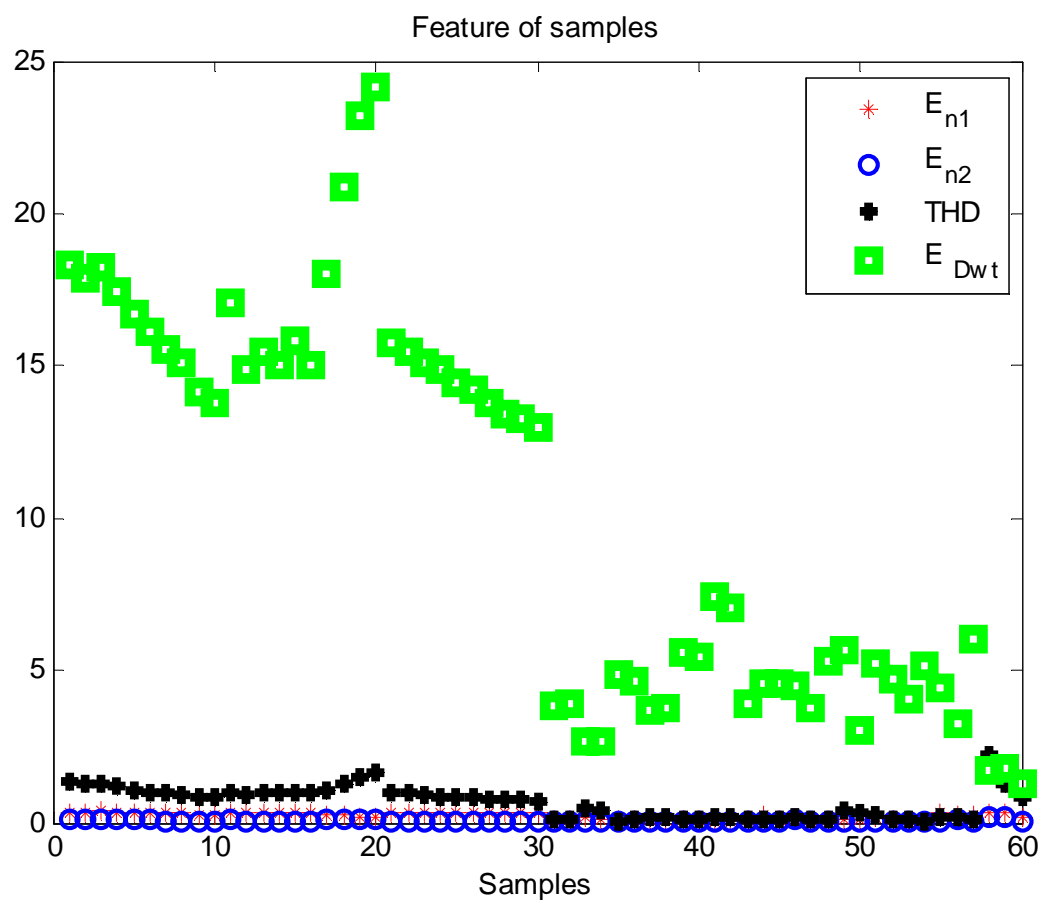

Fig. 8: The illustration of extracted features for 60 samples (the first 30 samples are inrush current and the other 30 samples are faulted current)

\subsection{Decision tree learning}

The next step after determining the desired feature is clustering of these features. By considering the Figure 11, the clustering of mentioned features for the first 30 samples (inrush current) and the other 30 samples (faulted current) are shown by I and F respectively, as is presented in flowchart of Figure 9. 
Bulletin de la Société Royale des Sciences de Liège, Vol. 86, special edition, 2017, p. 726 - 738

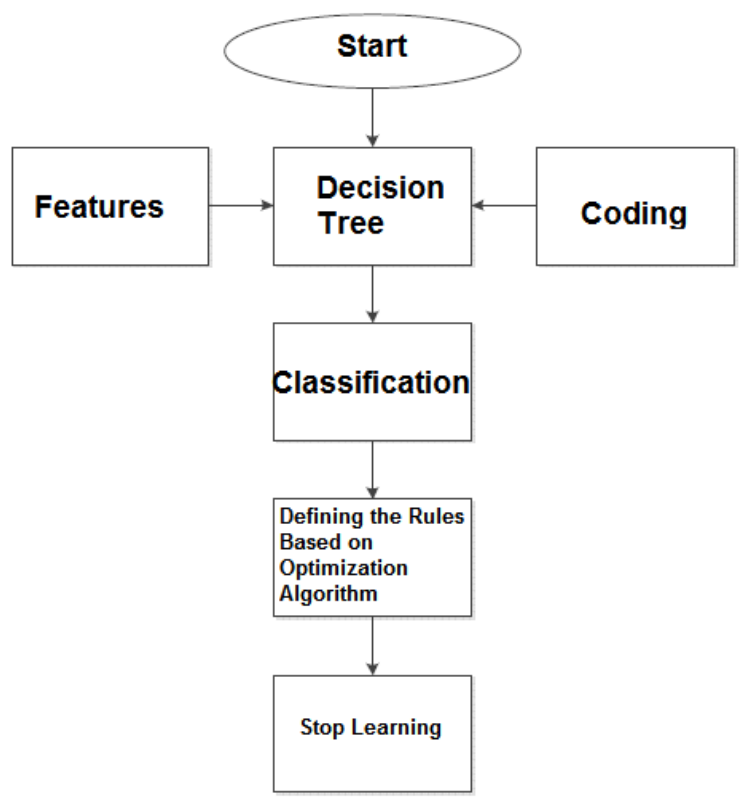

Fig. 9: The flowchart of tree learning

The considered samples in this paper contain the 30 internal faulted current samples (Figure 10) which is applied on the different phases and also 30 inrush current samples (Figure 11). The samples are extracted and simulated by PSCAD. In the following, the MATLAB is used to measure the signal in real environment in worst case by applying a $20 \mathrm{db}$ noise on signals and extract the mentioned features and create the decision tree and finally test it.
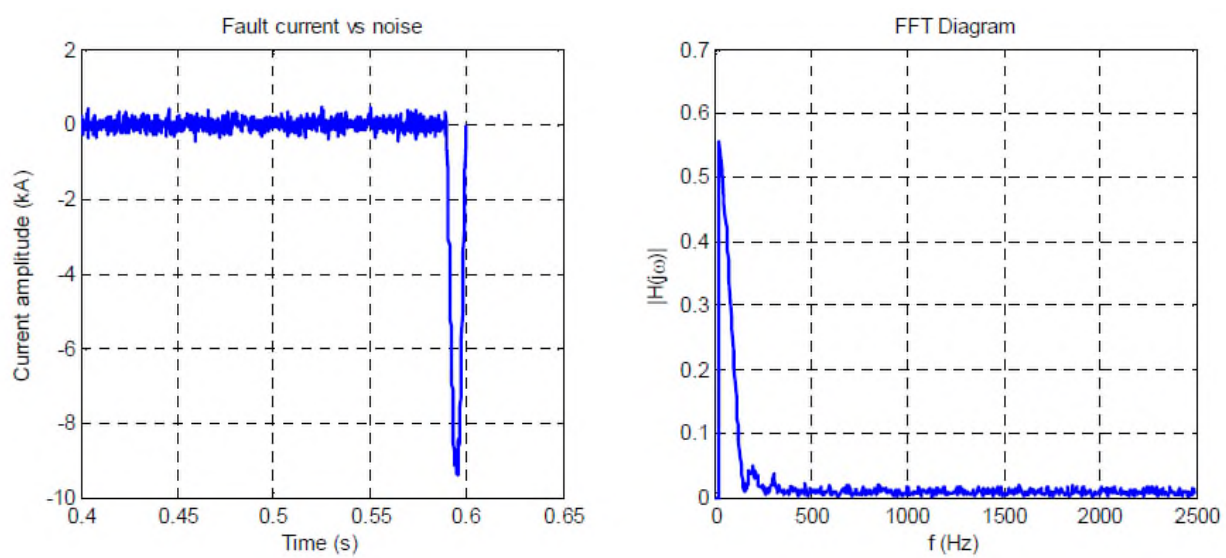

Fig. 10: A sample of faulted current by applying the $20 \mathrm{db}$ noise signal 

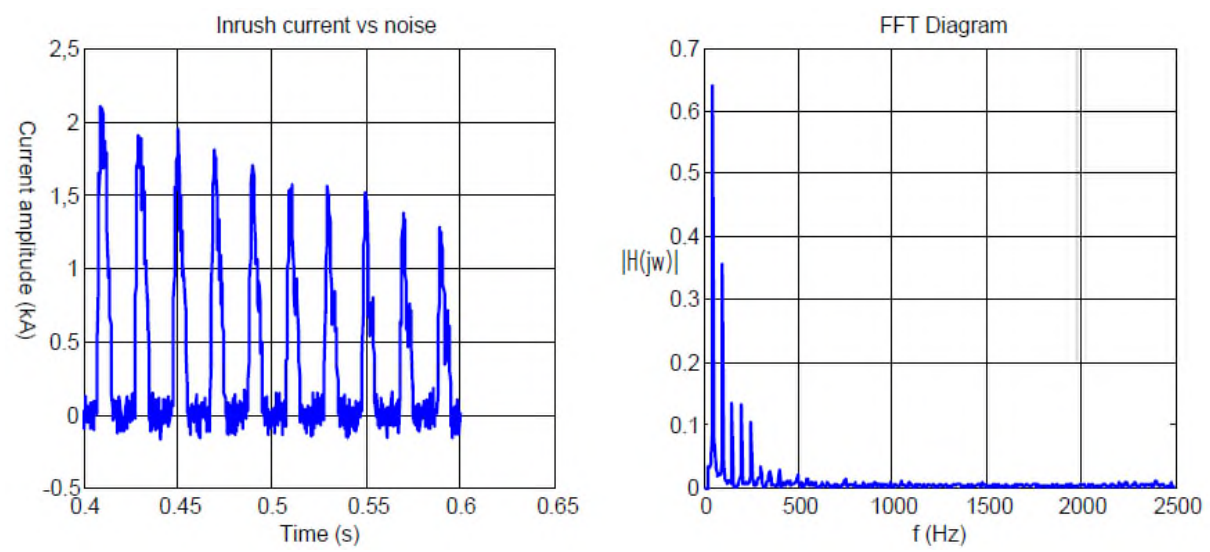

Fig. 11: A sample of inrush current by applying the $20 \mathrm{db}$ noise signal

Since the extracted features of the sample is different from the applied current, we can define an independent code for each current to clustering which leads to distinguish between faulted and inrush currents. After preparing the samples and extracting the features, the learning of the tree by a desired feature is started. The tree defines the clustering based on rules by receiving the features and dedicated code to each class based on optimization algorithms. These rules are eventual rules.

\subsection{Testing the tree in a system with stochastic and new samples}

The learned tree testing step is one of the most important steps since it explains the accurate learning and the performance of the tree. In this step, it must be chosen some currents stochastically and applied to the tree. If the tree could recognize these samples correctly, then we can conclude that tree learned completely and it can be applied to the relay as the making decision part. Otherwise the type of features or clustering method must be changed to obtain the 100 percent performance. As it mentioned in the previous steps, we must make sure about the effectiveness of the features before applying them to the tree so we can conclude that the feature of wavelet transform is been chosen properly.

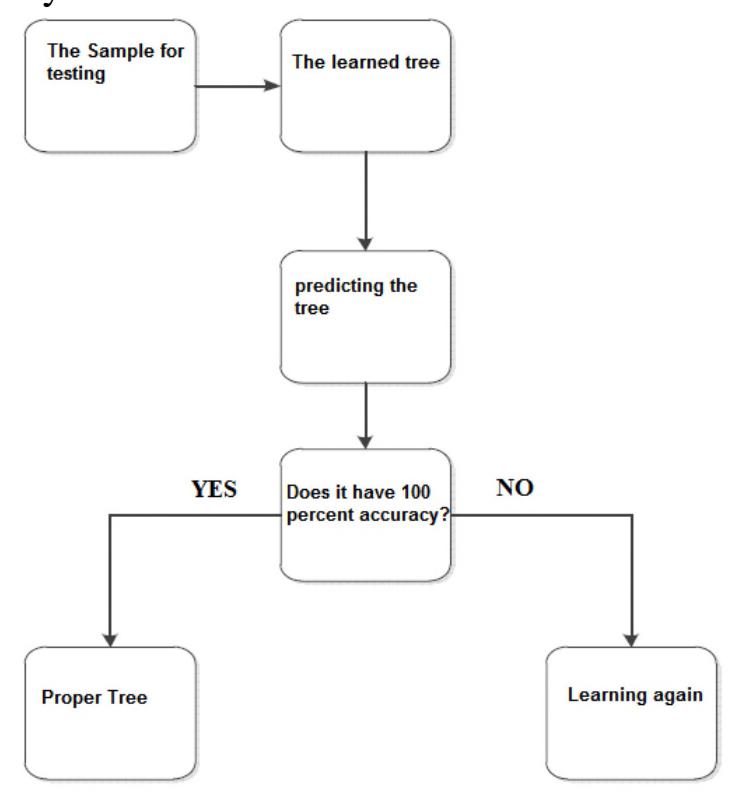

Fig. 12. The flowchart of testing the tree 


\section{The results of simuulation}

In this paper, the 5 faulted current and 5 inrush current samples are applied to the tree stochastically and testing the tree is accomplished in three ways. As it can be seen in Figure 13, the columns of the graph present that 10 new and stochastic samples are applied to the tree in different cases. In the first case (0) the learning level of the tree is measured by the primary samples and the testing of the second case (1) and third case (2) is done by means of clustering method and unknown, new and stochastic samples, respectively. As it is clear in Figure 13, the 100 percent accuracy is obtained in three cases.

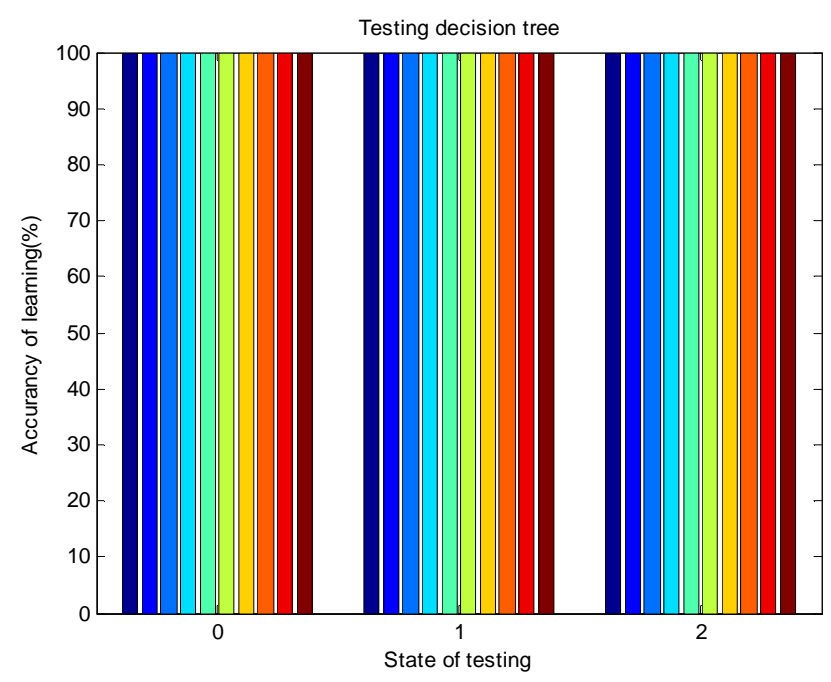

Fig. 13: Testing the tree in 3 cases for 10 samples

The presented method for differential protecting of the power transformer has some advantages compared to the current methods. Since the power quality events, this method has more flexibility than phase angle difference method in [4]. Moreover, the proposed method is faster than neural network and support vector machine methods in [5-7] due to using of the wavelet transform instead of discrete Fourier transform.

\section{Conclusion}

By considering that the mentioned relay has a proper performance by applying on the typical system in MATLAB/Simulink, we can use that as an appropriate industrial relay. This relay is suitable for Y-Y transformers since the used transforms. The designed relay in this paper has high speed and very good performance to protect the power transformers which it is very important. One of the characteristics of the designed relay is being adjustable the performance threshold of it in different industrial applications and distinguishing between the currents caused by saturating of the core of transformer and internal and external faults. This sometimes interfere the performance of the differential relay and it send the trip order incorrectly in some cases. On the other hand, it may occur an internal fault during the saturation of the transformer which this disturbance has minimum effect on the performance of the relay by analyzing the correlation coefficient. The minimum time of the fault detection from the beginning until reacting the relay is a few microseconds and its maximum time is less than 10 milliseconds which this feature provides high flexibility in various power systems contain of transformers and relay. 


\section{References}

[1] Rasoulpoor M, Banejad M. A correlation based method for discrimination between inrush and short circuit currents in differential protection of power transformer using Discrete Wavelet Transform: Theory, simulation and experimental Validation. Electrical Power and Energy Systems 2013; 51:168-77.

[2] Orlando Oliveira M, Suman Bretas A, Dornelles Ferreira G. Adaptive differential protection of three-phase power transformers based on transient signal analysis. Electrical Power and Energy Systems 2014; 57:366-74.

[3] Bejmert D, Rebizant W, Schiel 1. Transformer differential protection with fuzzy logic based inrush stabilization. Electrical Power and Energy Systems 2014; 63:51-63.

[4] Hosny A, K.Sood V. Transformer differential protection with phase angle difference based inrush restraint. Electric Power Systems Research 2014; 115:57-64.

[5] Ozgonenel O, Karagol S. Transformer differential protection using wavelet transform. Electric Power Systems Research 2014; 114:60-67.

[6] Geethanjali M, Mary Raja Slochanal S, Bhavani R. PSO trained ANN-based differential protection scheme for power transformers. Neurocomputing 2008; 71:904-18.

[7] Jazebi S, Vahidi B, Jannati M. A novel application of wavelet based SVM to transient phenomena identification of power transformers. Energy Conversation and Management 2011; 52:1354-63.

[8] Hossam Eldin A.A, Refaey M.A, A novel algorithm for discrimination between inrush current and internal faults in power transformer differential protection based on discrete wavelet transform. Electric Power Systems Research 2011; 81:19-24.

[9] Alencar R.J.N, Bezerra U.H, Ferreira A.M.D. A method to identify inrush currents in power transformers protection based on the differential current gradient. Electric Power Systems Research 2014; 111:78-84.

[10] Noshad B, Razaz M, Seifossadat S.G, A new algorithm based on Clarke's Transform and Discrete Wavelet Transform for the differential protection of three-phase power transformers considering the ultra-saturation phenomenon. Electric Power Systems Research 2014; 110:924.

[11] Yazdani-Asrami M, Taghipour-Gorjikolaie M, Razavi S.M, Gholamian S.A. A novel intelligent protection system for power transformers considering possible electrical faults, inrush current, CT saturation and over-excitation. Electrical Power and Energy Systems 2015; 64:1129-40.

[12] Tripathy M. Power transformer differential protection using neural network Principal Component Analysis and Radial Basis Function Neural Network. Simulation Modelling Practice and Theory 2010; 18:600-11.

[13] Oliveira L.M.R, Marques Cardoso A.J. Application of Park's power components to the differential protection of three-phase transformers. Electric Power Systems Research 2012; 83:203-11.

[14] Valsan S.P, Swarup K.S. Wavelet based transformer protection using high frequency power directional signals. Electric Power Systems Research 2008; 78:547-58.

[15] Monsef H, Lotfifard S. Internal fault current identification based on wavelet transform in power transformers. Electric Power Systems Research 2007; 77:1637-45. 
[16] Hamedani Golshan M.E, Saghaian-nejad M, Saha A, Samet H. A new method for recognizing internal faults from inrush current conditions in digital differential protection of power transformers. Electric Power Systems Research 2004; 71:61-71.

[17] Mohamed E.A, Abdelaziz A.Y, Mostafa A.S. A neural network-based scheme for fault diagnosis of power transformers. Electric Power Systems Research 2005; 75:29-39.

[18] Z. Moravej, D.N. Vishwakarma, S.P. Singh. Application of radial basis function neural network for differential relaying of a power transformer. Computers and Electrical Engineering 2003; 29:421-34.

[19] Samantaray S.R, Dash P.K. Decision Tree based discrimination between inrush currents and internal faults in power transformer. Electrical Power and Energy Systems 2011; 33:1043-48.

[20] Raisi, Z., Mohanna, F., \& Rezaei, M. (2011). Content-Based Image Retrieval for Tourism Application Using Handheld Devices". IJICTR Journal, 4, 57-64.

[21] Raisi, Zobeir, and Javad Azarakhsh. "Content Based Image Retrieval for Marine Life Images Using Ant Colony Optimization Feature Selection. 